\title{
Convergence analysis of cyclic iterative learning control scheme
}

DOI:

10.1109/IBCAST.2012.6177517

Link to publication record in Manchester Research Explorer

\section{Citation for published version (APA):}

Shaikh, I. U. H., Khalili, H. H., \& Brown, M. (2012). Convergence analysis of cyclic iterative learning control scheme. In Proceedings of 2012 9th International Bhurban Conference on Applied Sciences and Technology, IBCAST 2012/Proc. Int. Bhurban Conf. Appl. Sci. Technol., IBCAST (pp. 1-7). IEEE. https://doi.org/10.1109/IBCAST.2012.6177517

\section{Published in:}

Proceedings of 2012 9th International Bhurban Conference on Applied Sciences and Technology, IBCAST 2012|Proc. Int. Bhurban Conf. Appl. Sci. Technol., IBCAST

\section{Citing this paper}

Please note that where the full-text provided on Manchester Research Explorer is the Author Accepted Manuscript or Proof version this may differ from the final Published version. If citing, it is advised that you check and use the publisher's definitive version.

\section{General rights}

Copyright and moral rights for the publications made accessible in the Research Explorer are retained by the authors and/or other copyright owners and it is a condition of accessing publications that users recognise and abide by the legal requirements associated with these rights.

\section{Takedown policy}

If you believe that this document breaches copyright please refer to the University of Manchester's Takedown Procedures [http://man.ac.uk/04Y6Bo] or contact uml.scholarlycommunications@manchester.ac.uk providing relevant details, so we can investigate your claim.

\section{OPEN ACCESS}




\title{
Convergence Analysis of Cyclic Iterative Learning Control Scheme
}

\author{
Inam Ul Hasan Shaikh, Hassan H. Khalili, Martin Brown \\ Control Systems Centre, The University of Manchester, UK
}

\begin{abstract}
Iterative Learning Control (ILC) is a learning control technique for the systems operated repeatedly. The Iterative Learning Controller learns to generate the desired set of input signals to compensate for the output tracking errors. Conventionally the performance of ILC algorithms has been based on the convergence of the output tracking error. In this paper, the convergence of the control input is investigated down to the sample-time level. Two scenarios are considered: Firstly, when the control input is updated with same initial conditions at the start of each batch/repetition/iteration/trial and secondly for varying initial conditions. The batch to batch evolution of control inputs at each sample time within a batch is formulated. Convergence of the control input signals has been based on the Eigen analysis of this relationship. This provides deeper insight about the ILC algorithms and exact factors affecting the convergence could be monitored. Limits of the learning process are clearly demonstrated as well. Performance of Dtype \& PD-type ILC algorithms has been investigated for a simple pendulum and further extended to bipedal locomotion. Bipedal walking robot is an interesting control problem but involves complexity being a hybrid system. It comprises of single support, impact with ground and double support phases. The non-linear impacts pose challenge since they cause non-zero initial errors for each step. For reasons of energy efficiency, passive dynamics has been chosen for compass gait model of the biped. Stable gait achieved from a fine-tuned PD controller provides the set of desired inputs for the joints of the compass gait robot. ILC learns/adapts the joint control for repetitive gaits. It represents learning a sequence of action by muscles. Due to the transfer of state error in a cyclic manner from the end of a previous step/repetition to the recent step/repetition, the convergence has to be established in joint control and state space. The steady gait is achieved for bipedal locomotion on flat surface as demonstrated through simulations.
\end{abstract}

Keywords - Cyclic Iterative Learning Control, Convergence, bipedal walking robot

\section{INTRODUCTION}

Iterative Learning Control (ILC) is a promising area based on learning from past experience. Due to the repetitive nature of task for the robotic manipulators, ILC has been employed for enhanced tracking performance from its beginning in 1980's [1-4]. However, use of ILC to solve walking problem of the bipedal robot is relatively a new application area. Despite development of hardware for bipedal robots and advanced control techniques for walking, there are unsolved issues such as robustness, energy efficiency, complete autonomy, safety and userfriendliness for the bipedal robots. Bipedal robots cannot sustain large movements, variable speeds, etc. They also lack the level of robustness, versatility and adaptability that biological systems possess and use for efficient walking $[5,6]$. The control techniques for bipedal robot have been based on kinematic and dynamic modelling of the mechanism which requires complete state measurement and interaction between feet and ground. This requires heavy computation and optimization. Intelligent control techniques usually overcome these constraints [7]. As the biped robot has to act like a human, so it must be capable of learning the walking gaits from past experience as well like the humans. The learning ability allows the bipedal robot to modify its dynamic walking pattern to the changing conditions that is necessary for autonomous walking [8,9].

In ILC literature, the initial states which are the final states of the last repetition have been termed as "Cyclic learning control" [10, 11], "no-reset ILC (NRILC)" [12], 'continuous' ILC [13] or "alignment condition" [14]. A nonstandard approach of non-uniform repetition length was proposed in conjunction with PD-ILC to track periodic signals in non-linear systems [13]. In case of both the reference and the output trajectory following the "alignment condition", robust ILC algorithm based on the inverse dynamics of robotic manipulator was employed to achieve convergence [15]. Later, Lyapunov based ILC and RC algorithms were also formulated for robust convergence and evaluated the learning convergence rate $[14,16]$. The Lyapunov based ILC requires the knowledge of states which may not be available. Conditions for monotonic convergence were further formulated using PD-ILC with selective learning under restrictive assumptions for non-linear systems [17] and systems with non-parametric uncertainties [18].

Satoh et al. have used ILC to generate optimal walking gaits for one-legged hopping robot and further extended to biped on the basis of variational symmetry of the Hamiltonian systems [19, 20]. Iterative feedback tuning (IFT) has been employed in conjunction with ILC using virtual constraint to generate stable gait for compass gait robot [21]. Zhang et al. have proposed impulsive toe-off push generated with ILC which is applied to the biped just before the heel strike (i.e., the ground impact of swing leg). However, there is no actuation during the entire swing phase and the swing leg moves passively [22].

In bipedal walking the initial state for each step being a function of state at the end of last step is more complex than the generic "Cyclic ILC" or "alignment condition". This scenario has not been solved using ILC/RC techniques until now $[23,24]$. In this research, a new mathematical frame work for the two-dimensional evolution of input and state errors has been developed. The convergence of input and states for the "Cyclic" or "alignment" type initial state errors for D- and PD-ILC has been based on the rate of convergence analysis for zero initial errors [25]. Further, PD-ILC algorithm 
modified with the acceleration error term has been used to generate input torques at ankle and hip joint for the steady gait generation of compass gait model of the biped. The hybrid dynamics of compass gait biped locomotion are handled with the proposed scheme [26].

\section{ILC FOR PENDULUM}

\section{A. Damped Pendulum}

A simple damped pendulum in Fig. 1 is a widely studied control problem. It is selected here since corresponds to the swing leg of the robot. The D and PD-type ILC algorithm have been compared for learning performance to track the desired angle \& corresponding angular velocity by generating the desired control input signal at each sample time. The angle $\theta$ is measured anti clockwise. The control input torque $u$ is also in anti clockwise direction.

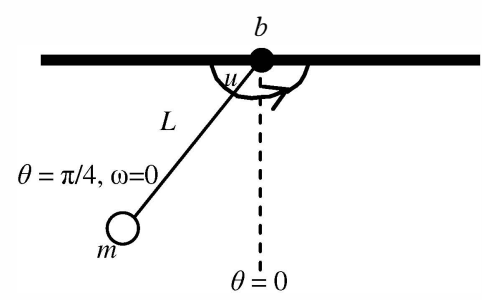

Figure 1 Damped pendulum

The pendulum is at rest with initial position $\theta=\pi / 4$ radians at the left side with initial angular velocity, $\omega=0 \mathrm{rad} / \mathrm{s}$. Each swing is motion of the pendulum from one extreme end to the other. The state space representation using state vector $\mathbf{x}=\left[\begin{array}{ll}\theta & \omega\end{array}\right]^{T}$ is given in Eq. (1).

$\dot{\mathbf{x}}=\left[\begin{array}{cc}0 & 1 \\ -g / L & -b / m L^{2}\end{array}\right] \mathbf{x}+\left[\begin{array}{c}0 \\ 1 / m L^{2}\end{array}\right] u$

where $L$ is the length, $m$ is mass, $g$ is the acceleration due to gravity and $b$ the damping coefficient. $u$ is the input torque applied. The ILC has to learn to compensate for gravity and the damping and follow the reference trajectory at each sample time which has been obtained from a fine-tuned PD controller.

\section{B. D-ILC algorithm for control input update}

The $1^{\text {st }}$ order D - ILC algorithm has been used to generate the control input for the consecutive swing/iteration as the sum of input at each sample time plus the scaled derivative of (output/state) error as in Eq.(2).

$$
u(i, j+1)=u(i, j)+\mathbf{K}_{\boldsymbol{d}}\{e(i+1, j)-e(i, j)\}
$$

where $u(i, j)$ is the input at ith sample time in swing/iteration number $j, u(i, j+1)$ is the input at $i$ th time instance in next batch numbered $j+1 . \mathbf{K}_{\boldsymbol{d}}$ is the gain matrix of suitable dimension. $e(i, j)$ is the state error at $i$ th sample time in batch no. $j$. The derivative of error has been approximated as forward difference.

The errors in the angle $\&$ the angular velocity at the last sample time of each swing are retained and fed back as the initial errors for the next swing as described by Eq.(3)

$$
\left[\begin{array}{c}
\Delta \theta(1, j+1) \\
\Delta \omega(1, j+1)
\end{array}\right]=\Phi\left[\begin{array}{l}
\Delta \theta(T, j) \\
\Delta \omega(T, j)
\end{array}\right]
$$

Here, $\Phi$ is the unity impact matrix for the alignment condition for the pendulum. It shall have different structure for walking robot discussed later.

\section{Convergence Analysis for D-ILC algorithm}

For the rate analysis of control input errors, the control input errors are augmented with initial state error in Eq. (3). Thus an augmented state error vector $\Delta \mathbf{x}^{*}(j)$ is formed in Eq. (4) below.

$$
\Delta \mathbf{x}^{*}(j)=[\Delta \theta(1, j), \Delta \mathrm{d} \theta(1, j), \Delta u(1, j), \Delta u(2, j), \ldots, \Delta u(M, j)](4)
$$

The evolution of the augmented state vector $\Delta \mathbf{x}^{*}(j)$ is governed by following matrix relationship:

$$
\Delta \mathbf{x}^{*}(j+1)=\mathbf{H}_{D}^{*} \boldsymbol{\Delta} \mathbf{x}^{*}(j)
$$

Where $\mathbf{H}_{D}^{*}$ in Eq. (6) is the operator matrix which relates the errors in the initial state \& control input at iteration $j$ with the next consecutive iteration $j+1$.

$$
\mathbf{H}_{D}^{*}=\left[\begin{array}{cccc}
\Phi \mathbf{A}^{M} & \Phi \mathbf{A}^{M-1} \mathbf{B} & \cdots & \Phi \mathbf{B} \\
\mathbf{K}_{d} \mathbf{C}(\mathbf{I}-\mathbf{A}) & \mathbf{I}-\mathbf{K}_{d} \mathbf{C B} & \mathbf{0} & \mathbf{0} \\
\mathbf{K}_{d} \mathbf{C A}(\mathbf{I}-\mathbf{A}) & \mathbf{K}_{d} \mathbf{C}(\mathbf{I}-\mathbf{A}) \mathbf{B} & \ddots & \vdots \\
\vdots & \vdots & \ddots & \mathbf{0} \\
\mathbf{K}_{d} \mathbf{C A}^{M-1}(\mathbf{I}-\mathbf{A}) & \mathbf{K}_{d} \mathbf{C A}^{M-2}(\mathbf{I}-\mathbf{A}) \mathbf{B} & \cdots & \mathbf{I}-\mathbf{K}_{d} \mathbf{C B}
\end{array}\right]
$$

It has been observed from simulations that D - type ILC algorithm converges for angular velocity only. A constant off-set error is maintained for the control input and angle. The persistence bounded state error indicates that magnitude of the largest eigenvalue of matrix $\mathbf{H}_{D}^{*}$ is equal to 1 , i.e. spectral radius lies at stability boundary. This observation leads to following theorem.

\section{Theorem:}

For a linear homogeneous system in Eq. (5), the operator matrix $\mathbf{H}_{D}^{*}$ as per Eq. (6) relates the evolution of errors in the initial state and control inputs obtained via Dtype ILC algorithm. The convergence is restricted due to the presence of the maximum Eigen value of $\mathbf{H}_{D}^{*}$ at the stability boundary.

Proof:

For any Eigen pair $\left(\lambda_{i}, \mathbf{v}_{i}\right)$ belonging to matrix $\mathbf{H}_{D}^{*}$ we have relation in Eq. (7) below.

$$
\left(\lambda_{i} \mathbf{I}-\mathbf{H}_{D}^{*}\right) \mathbf{v}_{i}=\mathbf{0}
$$

Denoting the eigenvector associated with unity Eigen value as $\mathbf{v}^{*}$, we get Eq. (8). 


$$
\begin{gathered}
\left(\mathbf{I}-\mathbf{H}_{D}^{*}\right) \mathbf{v}^{*}=\mathbf{0} \Rightarrow \\
{\left[\begin{array}{cccc}
\mathbf{I}-\mathbf{A}^{M} & -\mathbf{A}^{M-1} \mathbf{B} & \cdots & -\mathbf{B} \\
-\mathbf{K C}(\mathbf{I}-\mathbf{A}) & \mathbf{K C B} & \mathbf{0} & \mathbf{0} \\
-\mathbf{K C A}(\mathbf{I}-\mathbf{A}) & -\mathbf{K C}(\mathbf{I}-\mathbf{A}) \mathbf{B} & \ddots & \vdots \\
\vdots & \vdots & \ddots & \mathbf{0} \\
-\mathbf{K C A}^{M-1}(\mathbf{I}-\mathbf{A}) & -\mathbf{K C A}^{M-2}(\mathbf{I}-\mathbf{A}) \mathbf{B} & \cdots & \mathbf{K C B}
\end{array}\right] \times\left[\begin{array}{c}
v_{1}^{*} \\
v_{2}^{*} \\
v_{3}^{*} \\
v_{4}^{*} \\
\vdots \\
v_{M+2}^{*}
\end{array}\right]=\mathbf{0}}
\end{gathered}
$$

We can find the elements of the eigenvector $\mathbf{v}^{*}$ from individual rows. The $1^{\text {st }}$ block row as well as the $1^{\text {st }}$ block column is associated with two elements $v_{1}^{*}$ and $v_{2}^{*}$ corresponding to errors in angle $\&$ angular velocity respectively. Other rows are associated with single elements only which correspond to the errors in control input at each sampling instance. From $2^{\text {nd }}$ block row we get:

$$
\begin{aligned}
& -\mathbf{K C}(\mathbf{I}-\mathbf{A}) \times\left[v_{1}^{*}, v_{2}^{*}\right]^{\prime}+\mathbf{K C B} v_{3}^{*}=\mathbf{0} \\
& \Rightarrow\left[v_{1}^{*}, v_{2}^{*}\right]^{\prime}=(\mathbf{I}-\mathbf{A})^{-1} \mathbf{B} v_{3}^{*}
\end{aligned}
$$

From $3^{\text {rd }}$ block row we get.

$$
\begin{aligned}
& -\mathbf{K C A}(\mathbf{I}-\mathbf{A}) \times\left[v_{1}^{*}, v_{2}^{*}\right]^{\prime}-\mathbf{K C}(\mathbf{I}-\mathbf{A}) \mathbf{B} v_{3}^{*}+\mathbf{K C B} v_{4}^{*}=\mathbf{0} \\
& \Rightarrow \mathbf{A}(\mathbf{I}-\mathbf{A}) \times\left[v_{1}^{*}, v_{2}^{*}\right]^{\prime}+(\mathbf{I}-\mathbf{A}) \mathbf{B} v_{3}^{*}=\mathbf{B} v_{4}^{*}
\end{aligned}
$$

Using $\left[v_{1}^{*}, v_{2}^{*}\right]^{T}$ from Eq. (9), Eq. (10) is written as:

$$
\begin{aligned}
& \mathbf{A B} v_{3}^{*}+(\mathbf{I}-\mathbf{A}) \mathbf{B} v_{3}^{*}=\mathbf{B} v_{4}^{*} \\
& \Rightarrow \mathbf{A B} v_{3}^{*}+\mathbf{B} v_{3}^{*}-\mathbf{A B} v_{3}^{*}=\mathbf{B} v_{4}^{*} \\
& \Rightarrow v_{4}^{*}=v_{3}^{*}
\end{aligned}
$$$$
\text { Similarly, from } 4^{\text {th }} \text { block row we get Eq. }
$$$$
v_{5}^{*}=v_{3}^{*}
$$

So on and at the last row we have:

$$
v_{M+2}^{*}=v_{3}^{*}
$$

Hence, finally the dominant eigenvector $\mathbf{v}^{*}$ containing $M+2$ elements associated with unity Eigen value in terms of $v_{3}^{*}$ is given in Eq. (14).

$$
\mathbf{v}^{*}=v_{3}^{*} \times\left[(\mathbf{I}-\mathbf{A})^{-1} \mathbf{B}, 1,1, \ldots, 1\right]^{M}
$$

It is pointed that $1^{\text {st }}$ two elements of dominant eigenvector $\mathbf{v}^{*}$ correspond to the initial errors in angle \& angular velocity. The remaining $M$ elements which correspond to the errors in control input are all same. This indicates that when the solution converges to the dominant eigenvector after a number of iterations/swings then all the components corresponding to input errors have aligned in such a way that these have same values. It is thus the condition when D-ILC algorithm cannot update the control inputs any further because the state error used to update the control input has become constant.

\section{PD-ILC algorithm for control input update}

To achieve convergence for the input and angle along with the velocity for the damped pendulum, PD - ILC is employed. Adding the proportional error term scaled by gain matrix $\mathbf{K}_{p}$ in Eq. (2), the control input is updated as in Eq. (15) below.

$$
u(i, j+1)=u(i, j)+\mathbf{K}_{p} e(i+1, j)+\mathbf{K}_{d}\{\boldsymbol{e}(i+1, j)-\boldsymbol{e}(i, j)\}
$$

Using PD - ILC the augmented matrix $\mathbf{H}_{D}^{*}$ is amended as $\mathbf{H}_{P D}^{*}$ in Eq. (16) below.

$$
\mathbf{H}_{P D}^{*}=\left[\begin{array}{cccc}
\Phi \mathbf{A}^{M} & \Phi \mathbf{A}^{M-1} \mathbf{B} & \cdots & \Phi \mathbf{B} \\
\mathbf{K}_{d} \mathbf{C}(\mathbf{I}-\mathbf{A})-\mathbf{K}_{p} \mathbf{C} & \mathbf{I}-\mathbf{K}_{d} \mathbf{C} \mathbf{B} & \mathbf{0} & \mathbf{0} \\
\vdots & \ddots & \ddots & \vdots \\
\mathbf{K}_{d} \mathbf{C ~ A} \mathbf{A}^{M-1}(\mathbf{I}-\mathbf{A})-\mathbf{K}_{p} \mathbf{C} \mathbf{A}^{M-1} & \cdots & \cdots & \mathbf{I}-\mathbf{K}_{d} \mathbf{C ~ B}
\end{array}\right]
$$

The convergence occurs for selected values of $\mathbf{K}_{p}$ and $\mathbf{K}_{d}$ gain matrices for which all the Eigen values of matrix $\mathbf{H}_{P D}^{*}$ lie inside the unit circle.

\section{E. Simulations for Damped Pendulum}

The damped pendulum has been simulated to analyse the errors and rate of convergence for the initial state, control input $\&$ outputs over a number of swings/iterations for the "alignment condition". Each iteration/swing consists of 20 samples. Only the initial condition for the $1^{\text {st }}$ iteration is set same as that of desired. The control input during entire $1^{\text {st }}$ swing is zero. The pendulum has parameters:

$$
L=1 \mathrm{~m}, m=0.5 \mathrm{Kg}, g=9.81 \mathrm{~m} / \mathrm{s}^{2}, b=0.25 \mathrm{~N}-\mathrm{s} / \mathrm{m} \text {. }
$$

The linearised discrete space state matrices for the pendulum, sampled at 0.05 seconds are given in Eq. (17).

$$
\begin{array}{ll}
\mathbf{A}=\left[\begin{array}{cc}
0.9879 & 0.0492 \\
-0.4824 & 0.9633
\end{array}\right], \quad \mathbf{B}=\left[\begin{array}{l}
0.0012 \\
0.0492
\end{array}\right], \\
\mathbf{C}=\left[\begin{array}{ll}
1 & 0 \\
0 & 1
\end{array}\right], \quad \mathbf{D}=\left[\begin{array}{l}
0 \\
0
\end{array}\right]
\end{array}
$$

The time period for the pendulum is 2 seconds, so there are 20 samples for each swing from left to right and vice versa.

\section{a) D-ILC for damped pendulum}

For gain matrix $\mathbf{K}_{d}=[0.5,0.5]$, both the RMS errors in the initial state $\&$ input are minimised for the earlier 200 iterations in Fig. 2. The augmented initial state \& input error vector aligns with the dominant eigenvector at the rate $\left|\lambda_{2} / \lambda_{1}\right|$ where, $\lambda_{1} \& \lambda_{2}$ are the $1^{\text {st }}$ and $2^{\text {nd }}$ dominant Eigen values respectively.
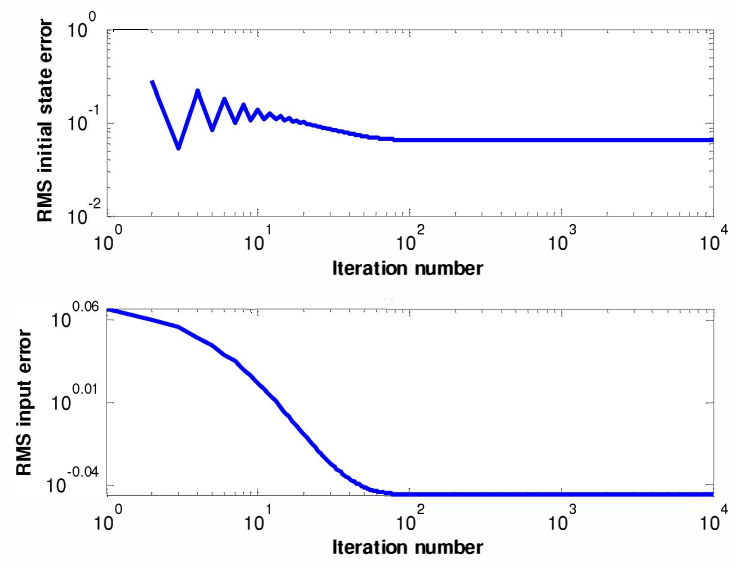

Figure 2 RMS values using D-ILC 


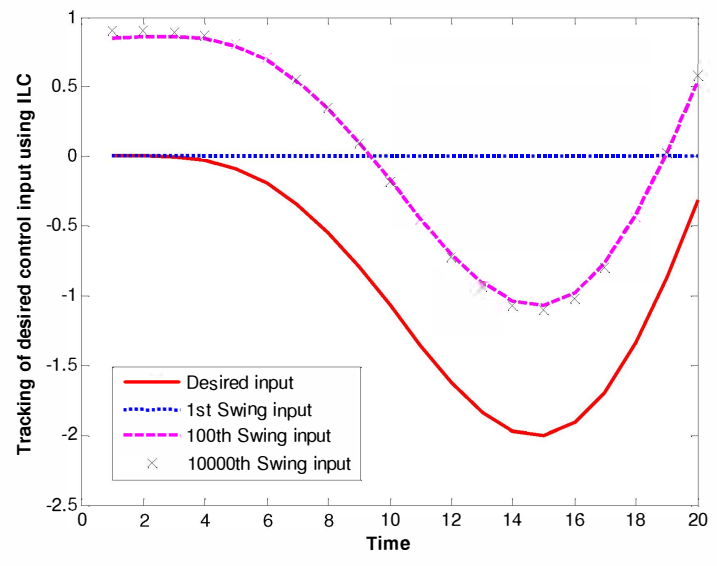

Figure 3 Tracking of control input using D-ILC
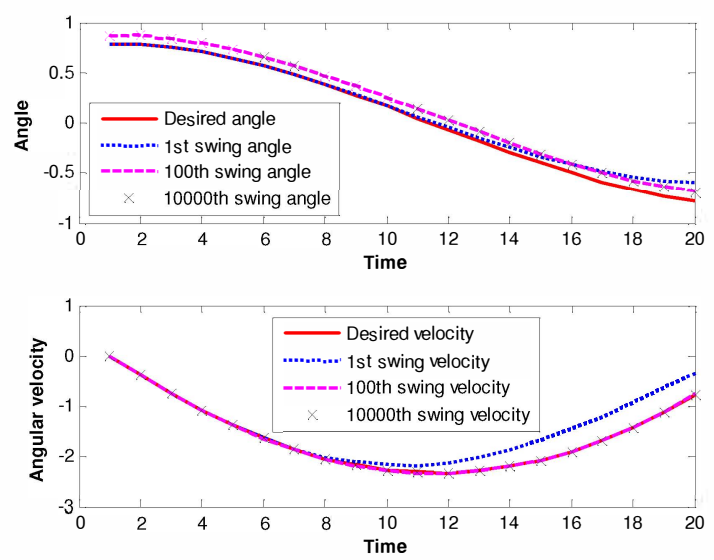

Figure 4 Tracking of angle \& velocity using D-ILC

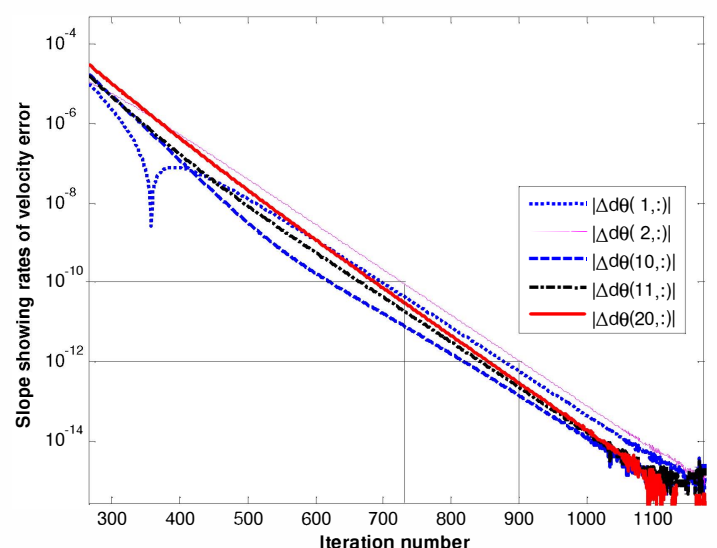

Figure 5 Convergence of velocity error using D-ILC

The velocity has converged but a constant off-set is observed in tracking of both the input and angle (Fig. 3 \& 4 respectively). The velocity has long-term convergence at a rate which equals the $2^{\text {nd }}$ dominant Eigen value 0.9749 . This corresponds to about 200 iterations for the decay of 0.01 in Fig. 5.

\section{b) PD-ILC for damped pendulum}

PD - ILC successfully minimises constant off-set errors in the input and angle along with the convergence of angular velocity. Using $\mathbf{K}_{p}=[0.5,0.5]$ and $\mathbf{K}_{d}=[0.5,0.5]$, the initial state errors and the input errors are minimised to $1.6184 \mathrm{e}-016$ and $3.8579 \mathrm{e}-015$ respectively (Fig. 6). From Fig. $7 \& 8$ it is observed that desired trajectories for all the three variables. i.e., angle, velocity and control input have been successfully tracked. The control input errors reach the numerical zero after 6000 iterations in Fig. 9. The maximum absolute Eigen value of the matrix $\mathbf{H}_{P D}^{*}$ is 0.9944 which indicates the maximum rate for control input error.
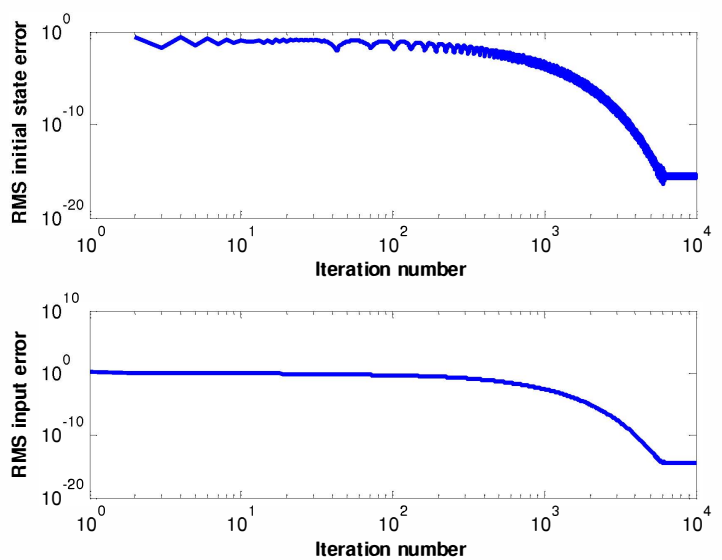

Figure 6 RMS values using PD-ILC

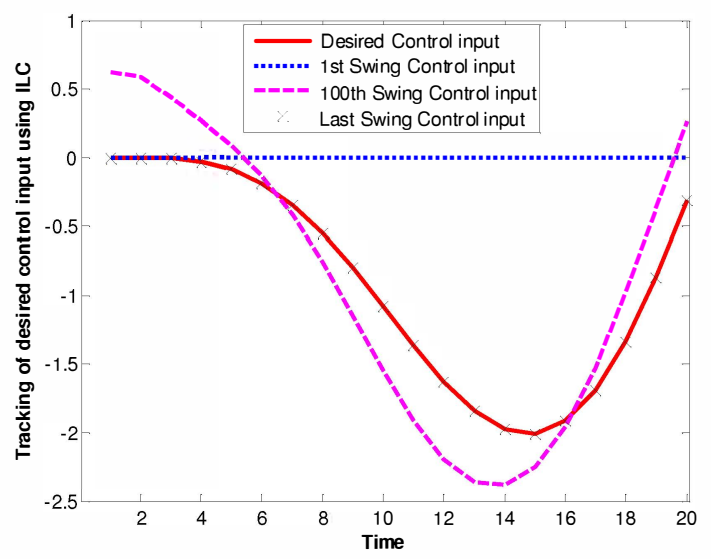

Figure 7 Tracking of control input using PD-ILC
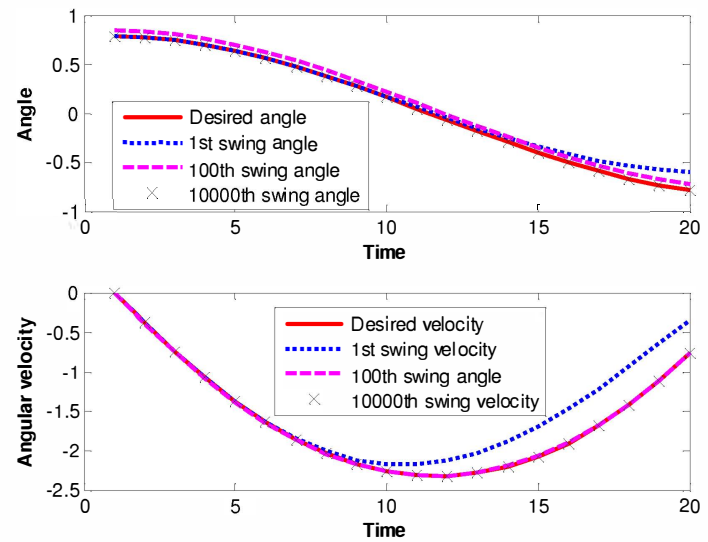

Figure 8 Tracking of angle \& velocity using PD-ILC 


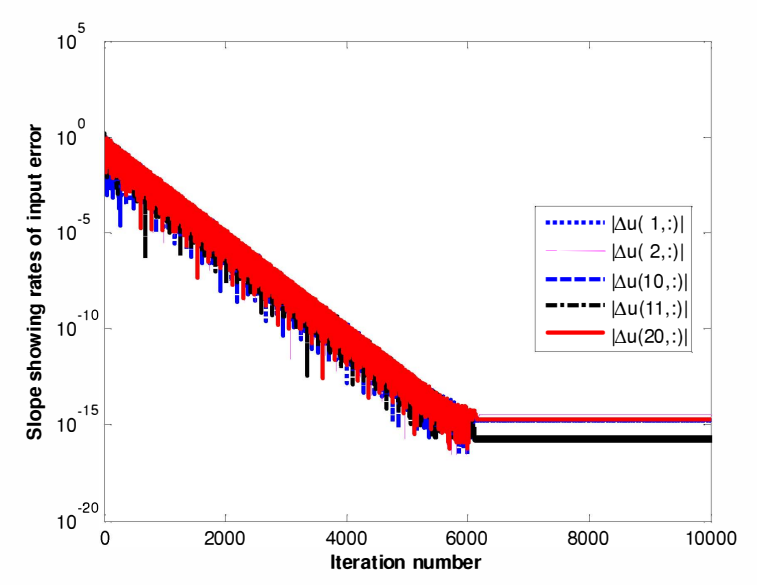

Figure 9 Convergence of input error using PD-ILC

\section{ILC FOR BIPEDAL LOCOMOTION}

Bipedal locomotion or walking with two feet consists of swinging motion by one leg while the other rotates around the ankle while its foot remains fixed which is called the stance foot. The impact occurs when the swing leg touches the ground. The change over of swing leg to stance leg and vice versa occurs. The new swing leg swings \& impacts with ground again. Each cycle/repetition constitutes a step for the biped [9].

ILC can be employed to generate input torques for the joints. At impact of the swing leg with ground the step swapping occurs but the state is not inherited exactly as in "Cyclic/Alignment ILC" discussed earlier. The joint angles and velocities at the start of new step are some function of those at the end of the previous step. Hence it is a special case of "Cyclic/Alignment ILC", since the initial conditions are different for each step until the desired steady state gait is achieved.

\section{A. Compass Gait robot}

Compass gait robot is the simplest model of biped used to study the nonlinear dynamics of the biped [27]. The two legs of the compass gait robot have no knees or feet and are connected with each other by a friction-less hip joint (Fig. 10). Here energy loss occurs due to ground impacts. Hence, in controller design the emphasis is on energy efficiency.

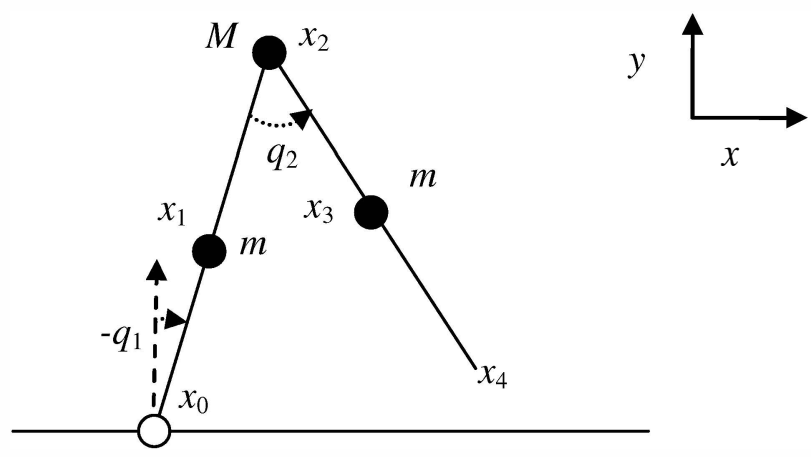

Figure 10 Compass gait robot

During the swing phase, the dynamics are given by the $2^{\text {nd }}$ order non-linear differential Eq. (18)

$$
\mathbf{M}(\mathbf{q}) \ddot{\mathbf{q}}+\mathbf{C}(\mathbf{q}, \dot{\mathbf{q}}) \dot{\mathbf{q}}+\mathbf{g}(\mathbf{q})=\left[\begin{array}{cc}
-1 & 0 \\
0 & 1
\end{array}\right]\left[\begin{array}{l}
\tau_{1} \\
\tau_{2}
\end{array}\right]=\mathbf{S u}
$$

where, vectors $\quad \mathbf{q}=[q 1, q 2]^{T} \quad$ and $\quad \dot{\mathbf{q}}=[\dot{q} 1, \dot{q} 2]^{T}$ represent the joint angles and the angular velocities respectively. $\mathbf{M}(\mathbf{q})$ is the inertia matrix, $\mathbf{C}(\mathbf{q}, \dot{\mathbf{q}})$ is the centripetal/coriolis matrix, and $\mathbf{g}(\mathbf{q})$ is the vector of gravity torques. The ankle joint actuator generates torque $\tau_{1}$ for the rotating the stance leg around the ankle joint. The ankle joint torque $\tau_{1}$ has negative multiplier -1 in selection matrix $\mathbf{S}$ to refer that it acts in (opposite) clockwise direction. The hip joint actuator applies torque $\tau_{2}$ to move forward the free leg so that the compass gait robot moves forward from left to right. Using $\mathbf{x}=[\mathbf{q}, \dot{\mathbf{q}}]^{T}$ as state, Eq. (19), gives the state space description of the compass gait.

$$
\dot{\mathbf{x}}=\left[\begin{array}{c}
\dot{\mathbf{q}} \\
-(\mathbf{M}(\mathbf{q}))^{-1}[\mathbf{C}(\mathbf{q}, \dot{\mathbf{q}}) \dot{\mathbf{q}}+\mathbf{g}(\mathbf{q})]
\end{array}\right]+\mathbf{S} \mathbf{u}
$$

When both feet are on the ground, it is double support phase. The swapping of feet occurs instantaneously assuming the angular momentum is conserved at the impact. The joint angles are reversed in direction and the joint velocities also change. The pre- $\&$ post- impact states (angles and velocities) are related as per Eq. (20).

$$
\left[\begin{array}{l}
\mathbf{q}^{+} \\
\dot{\mathbf{q}}^{+}
\end{array}\right]=\left[\begin{array}{cc}
-\mathbf{I} & \mathbf{0} \\
\mathbf{0} & \mathbf{R}
\end{array}\right]\left[\begin{array}{l}
\mathbf{q}^{-} \\
\dot{\mathbf{q}}^{-}
\end{array}\right]
$$

The superscripts - and + represent pre \& post impact values respectively. The matrix $\mathbf{R}$ is given in Eq. (21).

$\mathbf{R}=$

$$
\frac{\left[\begin{array}{cc}
-m+2(m+2 \times M) \cos (q 2) & -m \\
4(m+M)(\cos (2 \times q 2)-\cos (q 2)) & m(1-2 \cos (q 2))
\end{array}\right]}{4 M+3 m-2 m \cos (2 \times q 2)}
$$

where $m$ and $M$ are the leg and hip masses respectively and $q 2$ is the hip joint angle.

\section{B. Modified PD-ILC for the compass gait}

Following update algorithm based on PD-ILC has been employed.

$$
\begin{aligned}
u(i, j+1) & =u(i, j)+\mathbf{K}_{p} e(i+1, j) \\
& +\mathbf{K}_{d}\{e(i+1, j)-e(i, j)\}
\end{aligned}
$$

The gain matrix $\mathbf{K}_{d}$ has structure such that it utilizes forward difference errors of both the angle joints and their respective velocities. Effectively the acceleration term is also incorporated.

\section{Simulations for Compass Gait Robot}

The compass gait robot model has been discretized at sample time of 0.1 second. Reference trajectory for robot walking on flat surface has been obtained via a carefully tuned PD controller. The compass gait robot has been simulated for 4000 steps using gain matrices $\mathbf{K}_{p}=\left[\begin{array}{cccc}0.1 & 0 & 0 & 0 \\ 0 & 0.1 & 0 & 0\end{array}\right]$ and $\mathbf{K}_{d}=\left[\begin{array}{cccc}1 & 1 & 1 & 1 \\ 1 & 1 & 1 & 1\end{array}\right]$. 
The Iterative Learning Controller learns to generate the desired values of the ankle and hip joint torques as shown in Fig. 11 \& 12 respectively.

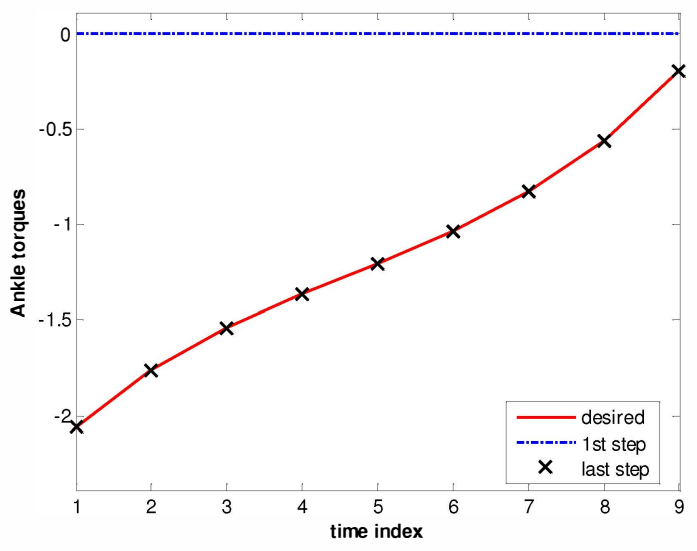

Figure 11 Tracking ankle joint torque

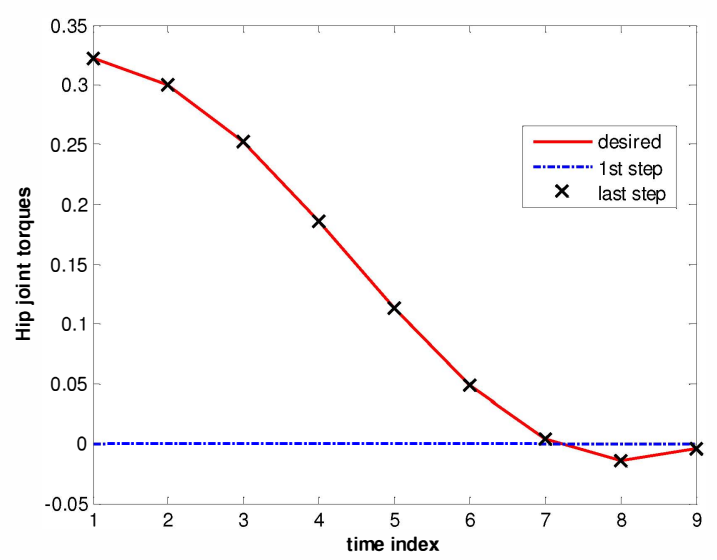

Figure 12 Tracking hip joint torque

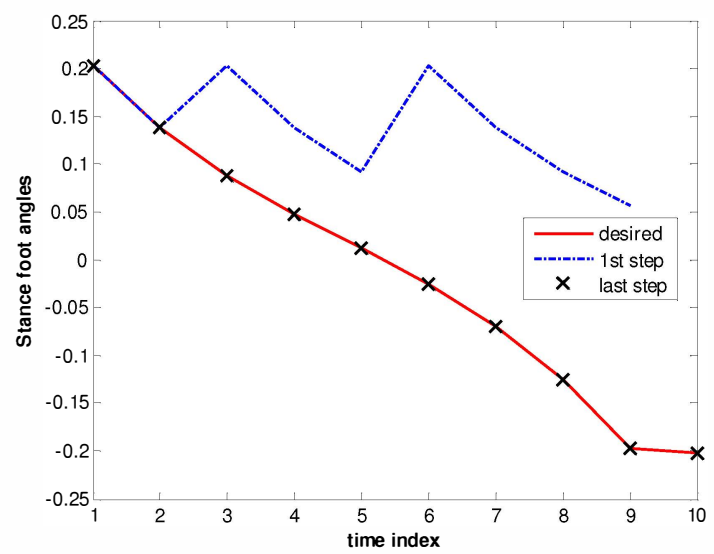

Figure 13 Tracking stance foot angle

As the joint input torques are tracked, the compass gait robot achieves a steady gait as observed from the angles of stance foot and hip joint in Fig. 13 and 14. The stable gait is also validated from the phase portraits in Fig. 15 \& 16 respectively.

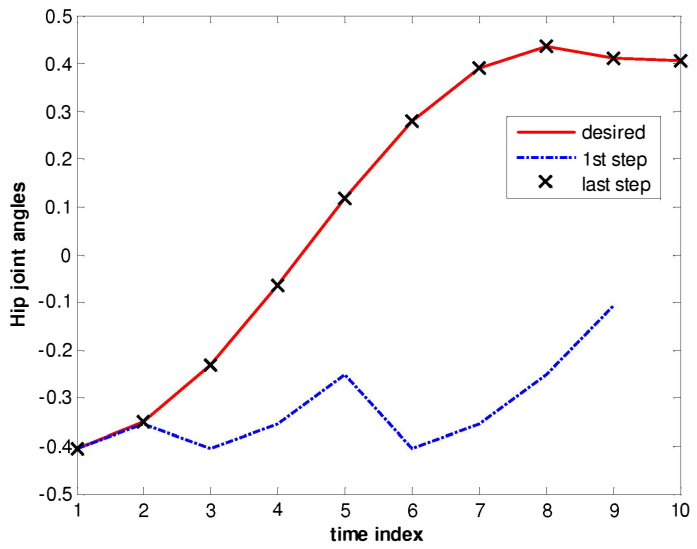

Figure 14 Tracking hip joint angle

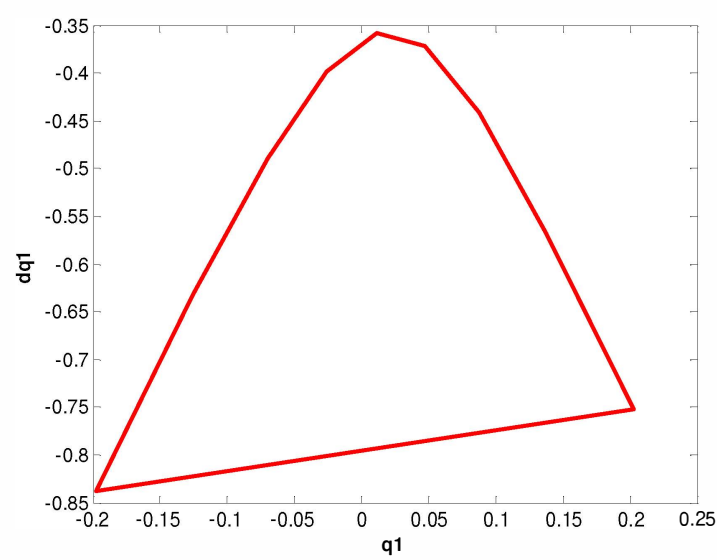

gure 15 Phase portrait of ankle joint

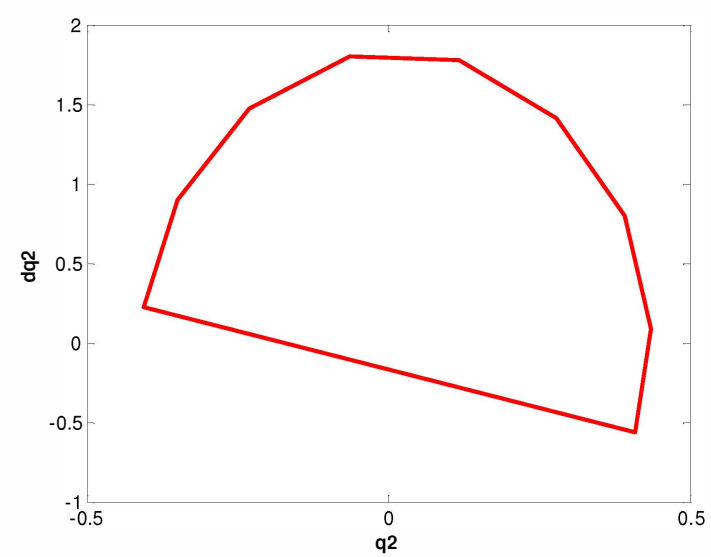

Figure 16 Phase portrait of hip joint

\section{CONCLUSIONS}

In this paper comparative analysis of $\mathrm{D}$ - and $\mathrm{PD}$ - type ILC algorithms has been presented for initial errors from previous iteration. The effect of eigenvalues of the matrix which relates the input error vectors has been investigated for damped pendulum. ILC has been further employed to solve bipedal walking problem. Stable gait has been achieved for the compass gait robot. Further topics under investigation include convergence \& robustness analysis of linearised compass gait robot with Iterative Learning Controller working together with stabilizing controllers. 


\section{ACKNOWLEDGMENT}

I. U. H. Shaikh thanks University of Engineering and Technology, Taxila and Higher Education Commission of Pakistan for the financial support.

\section{REFERENCES}

[1] Arimoto, S., S. Kawamura, and F. Miyazaki, Bettering operation of Robots by learning. Journal of Robotic Systems, 1984. 1(2): p. 123-140.

[2] Arimoto, S., S. Kawamura, and F. Miyazaki. Bettering operation of dynamic systems by learning: A new control theory for servomechanism or mechatronics systems. in The 23rd IEEE Conference on Decision and Control. 1984.

[3] Kawamura, S., F. Miyazaki, and S. Arimoto. Applications of learning method for dynamic control of robot manipulators. in 24th IEEE Conference on Decision and Control. 1985.

[4] Bristow, D.A., M. Tharayil, and A.G. Alleyne, A survey of iterative learning control. Control Systems Magazine, IEEE, 2006. 26(3): p. 96-114.

[5] Hurmuzlu, Y., F. Génot, and B. Brogliato, Modeling, stability and control of biped robots--a general framework. Automatica, 2004. 40(10): p. 1647-1664.

[6] Choi, B., ed. Humanoid Robots. 2009, In-Tech: Vienna, Austria.

[7] Vukobratovic, D.K.a.M., Humanoid Robots: New Developments, ed. A.C.d.P. Filho. 2007, Vienna, Austria I-Tech Education and Publishing.

[8] Chew, C.-M. and G.A. Pratt, Dynamic bipedal walking assisted by learning. Robotica, 2002. 20(05): p. 477-491.

[9] Kiriazov, P., Learning Robots to Walk Dynamically: biological control concepts, in 5th International Conference on Climbing and Walking Robots. 2002: Paris, France.

[10] Lucibello, P. and S. Panzieri. Cyclic control of linear systems: theory and experimental implementation on a flexible arm. in Decision and Control, 1994., Proceedings of the 33rd IEEE Conference on. 1994.

[11] Oriolo, G., S. Panzieri, and G. Ulivi. Cyclic learning control of chained-form systems with application to car-like robots. in 13th IFAC World Congress. 1996. San Francisco, CA, USA.

[12] Sison, L.G. and E.K.P. Chong. No-reset iterative learning control. in Proceedings of the 35th IEEE Decision and Control,. 1996.

[13] Moore, K.L., A non-standard iterative learning control approach to tracking periodic signals in discrete-time non-linear systems. International Journal of Control, 2000. 73(10): p. 955-967.

[14] Xu, J.-X. and R. Yan, On initial conditions in iterative learning control. IEEE Transactions on Automatic Control, 2005. 50(9): p. 1349-1354.
15] Xu, J.-X., B. Viswanathan, and Z. Qu, Robust learning control for robotic manipulators with an extension to a class of non-linear systems. International Journal of Control, 2000. 73(10): p. 858870.

[16] Mingxuan, S., S.S. Ge, and I.M.Y. Mareels, Adaptive repetitive learning control of robotic manipulators without the requirement for initial repositioning. IEEE Transactions on Robotics, 2006. 22(3): p. 563-568.

[17] Yang, Z. and C.W. Chan. An Iterative Learning Control with Alignment Initial Condition for a Class of Nonlinear Systems. in Chinese Control Conference, CCC. 2007.

[18] Yang, Z. and C.W. Chan, Conditional iterative learning control for non-linear systems with non-parametric uncertainties under alignment condition. IET Control Theory \& Applications, 2009. 3(11): p. 1521-1527.

[19] Satoh, S., K. Fujimoto, and S.H. Hyon. Gait Generation for Passive Running via Iterative Learning Control. in Intelligent Robots and Systems, 2006 IEEE/RSJ International Conference on. 2006.

[20] Satoh, S., K. Fujimoto, and S.-H. Hyon, Gait Generation for a Hopping Robot Via Iterative Learning Control Based on Variational Symmetry, in Lagrangian and Hamiltonian Methods for Nonlinear Control 2006, F. Bullo and K. Fujimoto, Editors. 2007, Springer Berlin / Heidelberg. p. 197-208.

[21] Satoh, S., K. Fujimoto, and S.H. Hyon. A framework for optimal gait generation via learning optimal control using virtual constraint. in IEEE/RSJ International Conference on Intelligent Robots and Systems, IROS. 2008.

[22] Qi-Zhi, Z., et al. Iterative learning control for biped walking. in International Conference on Mechatronics and Automation, ICMA. 2010.

[23] Wang, Y., F. Gao, and F.J. Doyle Iii, Survey on iterative learning control, repetitive control, and run-to-run control. Journal of Process Control, 2009. 19(10): p. 1589-1600.

[24] Owens, D., E. Rogers, and K.L. Moore, Analysis Of Linear Iterative Learning Control Schemes Using Repetitive Process Theory. Asian Journal of Control, 2002. 4(1): p. 68-89.

[25] Shaikh, I., Iterative learning controller - rate of convergence analysis, in PGR poster conference. 2010, The University of Manchester: Manchester, UK.

[26] Duindam, V. and S. Stramigioli, Modeling and Control for Efficient Bipedal Walking Robots - A Port-Based Approach. Springer Tracts in Advanced Robotics, ed. B. Siciliano, O. Khatib, and F. Groen. Vol. 53. 2009: Springer Berlin / Heidelberg.

[27] Golliday, C., Jr. and H. Hemami, An approach to analyzing biped locomotion dynamics and designing robot locomotion controls. IEEE Transactions on Automatic Control, 1977. 22(6): p. 963972. 